Review Article

\title{
Meta-Analysis of the Effect of Traditional Chinese Medicine Compounds Combined with Standard Western Medicine for the Treatment of Diabetes Mellitus Complicated by Coronary Heart Disease
}

\author{
Mengqi Yang $\mathbb{D},{ }^{1,2,3,4}$ Min Cheng, ${ }^{5}$ Min Wang $\mathbb{D},{ }^{6}$ Zhishu Tang $\mathbb{D},{ }^{1,2,3,4}$ Zhongxing Song, ${ }^{1,2,3,4}$ \\ Chunli Cui, ${ }^{1,2,3,4}$ Yanru Liu, ${ }^{1,2,3,4}$ and Zhen Zhang ${ }^{1,2,3,4}$ \\ ${ }^{1}$ Shaanxi University of Chinese Medicine, Xianyang 712046, China \\ ${ }^{2}$ Co-construction Collaborative Innovation Center for Chinese Medicine Resources Industrialization By Shaanxi and Education \\ Ministry, Xianyang 712046, China \\ ${ }^{3}$ State Key Laboratory of Research and Development of Characteristic Qin Medicine Resources (Cultivation), \\ Xianyang 712046, China \\ ${ }^{4}$ Chinese Medicine Industry Research Institute, Xianyang 712046, China \\ ${ }^{5}$ Shangluo University, Shangluo 726000, China \\ ${ }^{6}$ The Second Affiliated Hospital, Shaanxi University of Chinese Medicine, Xianyang 712046, China
}

Correspondence should be addressed to Min Wang; 156898938@qq.com and Zhishu Tang; tzs6565@163.com

Received 25 February 2021; Accepted 5 June 2021; Published 21 June 2021

Academic Editor: Francesca Mancianti

Copyright ( 2021 Mengqi Yang et al. This is an open access article distributed under the Creative Commons Attribution License, which permits unrestricted use, distribution, and reproduction in any medium, provided the original work is properly cited.

This study aimed to systematically evaluate the clinical efficacy and safety of traditional Chinese medicine (TCM) compounds combined with standard treatments for diabetes mellitus (DM) complicated by coronary heart disease (CHD). We performed a systematic and comprehensive search of the China Knowledge Network, WanFang, WeiPu, PubMed, and Web of Science, including Chinese and English articles, for randomized controlled trials (RCTs) assessing the use of Chinese herbal compounds for the treatment of DM complicated by CHD published before June 1,2020. The literature was screened according to standard criteria. Risk assessment, based on the Jadad scale, was performed using the Review Manager5.3 software for meta-analysis. In total, 23 articles were selected, including 2405 cases. The meta-analysis showed that the combination of standard treatments with TCM compounds significantly improved the overall treatment efficacy for DM complicated by $\mathrm{CHD}$ (OR(odds ratio) $=4.39$; 95\% confidence interval (95\% CI), 3.30-5.84; $P<0.0001$ ), fasting blood glucose level (mean difference (MD) $=-1.04 ; 95 \% \mathrm{CI},-1.96$ to $-0.13 ; P=0.03$ ), total cholesterol level $(\mathrm{MD}=-1.16 ; 95 \% \mathrm{CI},-1.48$ to $-0.83 ; P<0.0001)$, triglyceride $(\mathrm{MD}=-0.46 ; 95 \% \mathrm{CI},-0.62$ to $-0.29 ; P<0.0001)$, low-density lipoprotein level $(\mathrm{MD}=-0.57 ; 95 \% \mathrm{CI},-0.87$ to $-0.27 ; P=0.0002)$, high-density lipoprotein level $(\mathrm{MD}=0.19 ; 95 \% \mathrm{CI}$, 0.12 to $0.26 ; P=0.02)$, and electrocardiogram $(\mathrm{OR}=4.20 ; 95 \% \mathrm{CI}, 3.15$ to $8.18 ; P<0.0001)$. In contrast, there was no improvement of 2-hour postprandial glucose level $(\mathrm{MD}=-1.03 ; 95 \% \mathrm{CI},-2.14$ to $0.08 ; P=0.07)$, or adverse reactions $(\mathrm{OR}=0.53 ; 95 \% \mathrm{CI}, 0.19$ to 5.50; $P=0.21$ ). We concluded that the combined therapy has some benefits in treating DM complicated by CHD. However, these results should be confirmed by further referenced evidence, high risk assessment, and lower publication bias.

\section{Introduction}

Diabetes mellitus (DM) is a metabolic disorder characterized by hyperglycemia [1]. Its etiology is complex and related complications severely threaten human health. Both coronary heart disease (CHD) and DM are common clinical conditions. Coronary atherosclerotic heart disease is referred to as coronary artery disease, which leads to stenosis or occlusion of the artery lumen and eventually to myocardial ischemia [2]. Studies have shown that type 2 DM 
(T2DM) is associated with higher risk of CHD [3]. The pathological basis of CHD is myocardial ischemia, hypoxia, or necrosis caused by coronary atherosclerosis. DM is one of the risk factors for premature arteriosclerosis and can further contribute to the deterioration of CHD. Data showed that diabetic patients are four times more likely to develop cardiovascular disease than nondiabetic patients [4]. DM complicated by $\mathrm{CHD}$ often leads to multiple organ damage, seriously reducing the quality of life of the patients, and it can even be life threatening [5]. Clinical treatment of DM complicated by CHD mainly aims at improving myocardial blood supply and controlling blood glucose through the use of antiplatelet aggregation, anticoagulation, antiperoxidation, antihypertension, blood lipid regulation, thrombolysis, bypass grafting, and arterial interventional therapy. However, these strategies require long-term therapy, only have a single target, and associated with poor patient compliance with the drug regimen, adverse drug reactions, and poor prognosis, which leads to poor overall clinical efficacy [6]. In traditional Chinese medicine (TCM) theory, CHD and DM are classified as "chest arthralgia", "heartache," and "thirst elimination" [7].

The main clinical manifestation of $\mathrm{CHD}$ is angina pectoris, often accompanied by panic, shortness of breath, and fatigue. Ancient medical books recorded a lot of prescriptions for the treatment of chest arthralgia, including Wenyang Ton gmai, Yiqi Shengxue, Yangyin Shengmai, and other conventional prescriptions. TCM diagnosis criteria of DM is lung heat injury and fire-flourishing Yin deficiency, often calling for Xiaoke Fang and Qingwei San dialectical treatments [8]. Recently, the scientific literature reported that TCM compounds can exert certain therapeutic effects on DM and CHD [9]. A study by Zhang et al. [10] showed that Western medicine basic therapy supplemented by Yiqi Yangyin Huoxue decoction provides a better treatment for DM complicated by CHD than Western medicine used alone, and it could increase the curative effects of Western medicine. Another study by Zou $[11,12]$ showed that the addition of Yixinshu capsules can relieve symptoms in patients suffering from T2DM-related CHD angina pectoris, enhance exercise tolerance, reduce blood glucose level, and the capsule is safe, effective, and easily tolerated. Finally, addition of Pingxiaotongxin capsules added to standard treatment can also alleviate patient symptoms and reduce their blood glucose level. However, there is still insufficient evidence supporting the use of these TCM compounds. To fill this gap, we sought to provide statistics-based evidence as reference for the clinical application of TCM by performing a systematic meta-analysis comparing the efficacy of Western standard therapy used alone (control group) or in combination with TCM compounds (observation group) for the treatment of DM complicated by CHD.

\section{Methods}

2.1. Inclusion Criteria. The patients included in this study met the diagnostic criteria for CHD established by the Ministry of Health of the People's Republic of China and the diagnostic criteria for DM published by the World Health
Organization in 2019. Only publications in English or Chinese were included. The included study's indicators of efficacy included at least total efficiency or blood glucose levels.

2.2. Exclusion Criteria. Excluded documents included literature reviews, meta-analyses, studies in animal models, duplicate publication, and retrospective studies, such as clinical studies designed without a control group or case reports.

2.3. Intervention. For inclusion of a study, the control group had to be treated with standard Western medicine protocol, and the observation group had to be treated with the same standard Western medicine protocol as the control group, in combination with TCM compounds. The specific dosage and time of administration were not clearly defined.

2.4. Evaluation Indicators. The clinical indicators included total effective rate, levels of fasting blood glucose, postprandial 2-hour plasma glucose, total cholesterol, triglycerides, high-density lipoprotein cholesterol, and low-density lipoprotein cholesterol, as well as frequency of angina attacks and the duration of angina pectoris pain.

2.5. Search Strategies. We searched the China National Knowledge Infrastructure (CNKI), WanFang, the Cochrane Library, and CBM (documents collected from January 2000 to February 2020) on traditional Chinese medicine compounds combined with standard Western medicine for the treatment of DM complicated by coronary heart disease. The language of the text was limited to Chinese and English. Theme for database searching were "Chinese medicine compound," "DM with coronary heart disease," and "randomized control".

2.6. Data Extraction and Risk Assessment. In total, 23 articles were selected and cross-checked by two researchers. The controversial content or the score of the article was determined by one-third person after careful assessment. Collected information included the author names, year of publication, the numbers of cases, methodological characteristics, outcome measures, adverse events, and follow-up records. These data were separately collected and crosschecked by two researchers. The controversial content was determined by one-third person after careful assessment. Risk assessment was based on the Jadad scale. The specific points for the evaluation included randomization scheme (2 points), blind mode (2 points), and exit and missing visit (1 point). Based on the score, the articles were classified as low quality ( $0-2$ points), medium quality ( $3-4$ points), and high quality (5 points).

2.7. Statistical Analysis. The meta-analysis was performed using the Cochrane Collaboration's software RevMan 5.3. Heterogeneity was assessed by means of $I^{2}$ statistics. The 
fixed effect model was used to analyze data with low heterogeneity. The random effects model was used to analyze the data with $I^{2}>50 \%$, representing high heterogeneity. Outcome measures, such as total efficiency rate (TER) and adverse events (AEs) as dichotomous variables, were analyzed using odds ratio (OR) values with 95\% confidence interval $(95 \% \mathrm{CI})$. Continuous data are presented as weighted mean difference (MD) with a 95\% CI. Funnel plots were used to analyze publication bias.

\section{Results}

3.1. Literature Retrieval. In total, 23 randomized controlled trials (RCTs) were selected, including 2405 cases. The article screening process and results are shown in Figure 1.

3.2. Incorporation of the Basic Features of the Literature. Based on the above literature screening process, 23 RCTs were selected finally, gathering a total of 2405 samples, including 1219 were in the observation groups and 1186 were in the control groups. The demographic baseline indicators were not statistically different and comparable across the different studies. Specific information is reported in Table 1.

3.3. Document Evaluation. Based on the Jadad scale, the 23 articles were evaluated systematically from the aspects of "randomization mode," "blind method strategy," and "exit and missing visits." Regarding the randomization mode, in the studies, Ye [17], Li and Shang [31], Guan and Shang [19], Xing et al. [18], Zhang and Zhang [16], Yan et al. [28], He [32], and Sun et al. [24] used the random number table method to perform a random grouping, which corresponds to a standard grouping mode and gave a 2-point assignment. Zhang [15] used a random allocation method, Zhang [13] used a random group method, and Zhang [27] used a file sequence all corresponding to "pseudorandomization" methods and that gave 0-point assignment. In the other selected articles [14, 20-23, 25, 26, 29-31, 33-35], no randomization method was specified, and 0 point were assigned. None of the selected studies had implemented a blinding procedure, and therefore, all scored 0 . Studies that did not have any dropout or lost follow-up were assigned 1 point. Based on the above scoring, only 8 of the 23 selected articles reached a medium quality ( 3 points), whereas the other articles were of low quality (1 point). The overall article evidence level was low, and the error risk was high, as shown in Table 2.

\subsection{Meta-Analysis}

3.4.1. Total Clinical Efficiency. A total of 1785 participants were reported in 20 studies with no statistical between-study heterogeneity $\left(I^{2}=0 \% ; P=0.09\right)$. Therefore, a meta-analysis of the data from these 20 studies was performed according to the fixed effect model. The results of the comparison between standard treatments alone and standard treatment combined with TCM compounds showed that the TCM compounds improved the overall clinical efficiency of treatment for DM complicated by CHD $(\mathrm{OR}=4.20 ; 95 \% \mathrm{CI}$, 3.21-5.50; $P=0.0001$; Figure 2).

3.4.2. Fasting Blood Glucose (FBG) Level. The FBG levels were reported for a total of 832 participants across 11 studies that were statistically heterogeneous $\left(I^{2}=99 \% ; P<0.001\right)$. Therefore, the meta-analysis of the data was performed using the random effects model. The results showed that addition of TCM compounds to standard treatments reduced the FBG levels $(\mathrm{MD}=-1.02 ; 95 \% \mathrm{CI},-1.87$ to $-0.17 ; P=0.02$; Figure 3).

3.4.3. Two-Hour Plasma Glucose (2hPG). The $2 \mathrm{hPG}$ levels were reported for a total of 621 participants across 8 studies that presented with statistical between-study heterogeneity $\left(I^{2}=99 \% ; P<0.001\right)$. Therefore, the meta-analysis of the data was performed using the random effects model. The comparison between standard treatments alone and standard treatments combined with TCM compounds showed that the addition of the TCM compounds to the therapy had no effect on $2 \mathrm{hPG}$ levels $(\mathrm{MD}=-1.07 ; 95 \% \mathrm{CI},-2.09$ to $-0.08 ; P=0.04$; Figure 4).

3.4.4. Total Cholesterol. Total cholesterol levels were measured for a total of 1490 participants across 13 studies presented with statistical between-study heterogeneity $\left(I^{2}=98 \% ; P<0.001\right)$. Therefore, the meta-analysis of the data was performed using the random effects model. This analysis showed that TCM compounds combined with standard treatments ameliorated the total cholesterol levels of the patients suffering from DM complicated by $\mathrm{CHD}$ complications, compared with standard treatments used alone $(\mathrm{MD}=-1.16 ; 95 \% \mathrm{CI},-1.48$ to $-0.83 ; P=0.0001$; Figure 5).

3.4.5. Triglycerides. Triglyceride levels were reported for a total of 1374 participants in 12 studies. The statistical heterogeneity of the results from these studies $\left(I^{2}=94 \%\right.$; $P<0.001)$. Therefore, the meta-analysis of the data was performed using the random effects model. The addition of TCM compounds to standard treatments resulted in a beneficial effect on triglyceride levels $(\mathrm{MD}=-0.46 ; 95 \% \mathrm{CI}$, -0.62 to $-0.29 ; P=0.0001$; Figure 6 ).

3.4.6. Low-Density Lipoprotein (LDL-C). LDL-C levels were reported for a total of 1274 participants in 11studies. These studies had statistically heterogeneous results $\left(I^{2}=96 \%\right.$; $P<0.001)$. Therefore, the meta-analysis of the data was performed using the random effects model. The addition of the effect of TCM compounds to standard treatments resulted in a beneficial effect $(\mathrm{MD}=-0.57 ; 95 \% \mathrm{CI},-0.87$ to $-0.27 ; P=0.0002$; Figure 7 ).

3.4.7. High-Density Lipoprotein (HDL-C). HDL-C levels were reported for a total of 1374 participants in 12 studies 


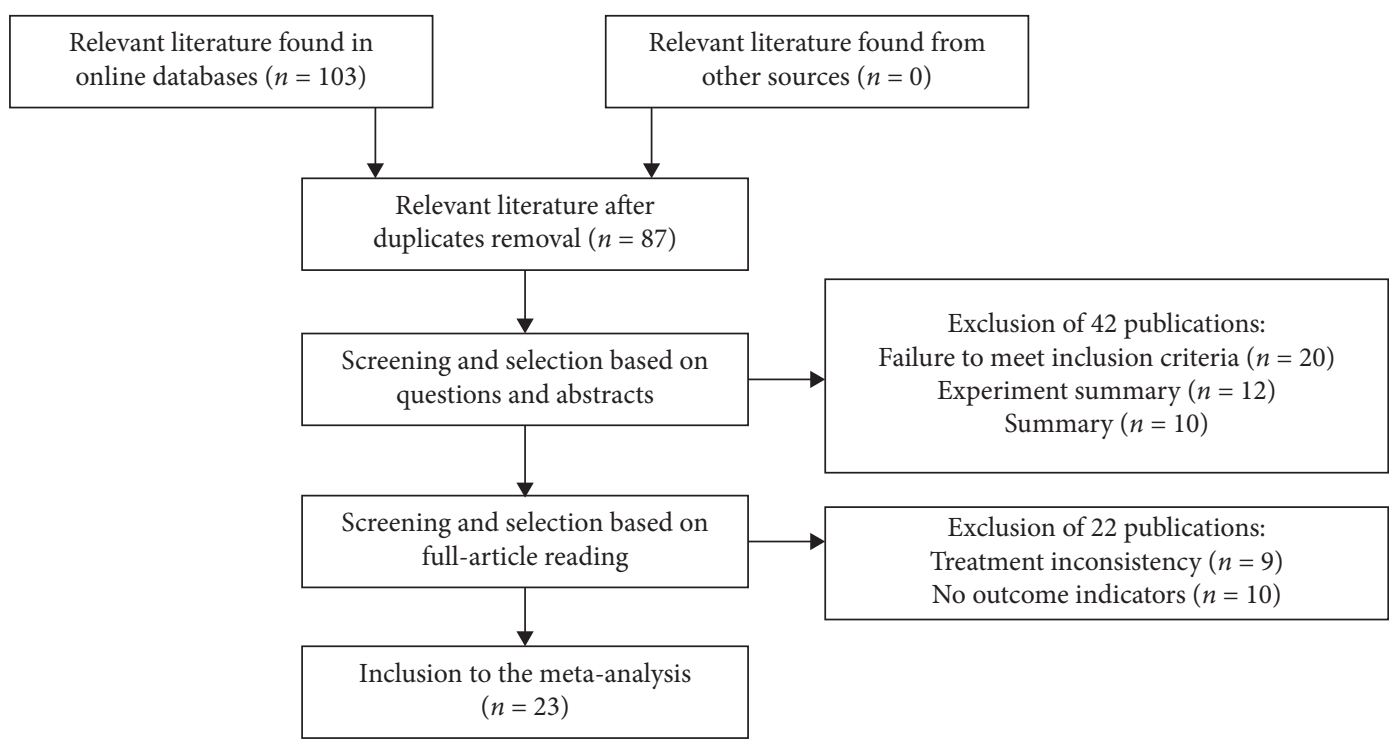

FIgURE 1: Search flow diagram.

TABLe 1: Basic characteristic of included studies.

\begin{tabular}{|c|c|c|c|c|c|c|c|c|c|c|}
\hline \multirow{2}{*}{$\begin{array}{l}\text { Inclusion } \\
\text { studies }\end{array}$} & \multicolumn{2}{|c|}{$\begin{array}{l}\text { Sample } \\
\text { size }\end{array}$} & \multicolumn{2}{|c|}{$\begin{array}{l}\text { Male/ } \\
\text { female }\end{array}$} & \multicolumn{2}{|c|}{ Average age } & \multicolumn{2}{|c|}{ Intervention measures } & \multirow{2}{*}{$\begin{array}{l}\text { Treatment } \\
\text { course }\end{array}$} & \multirow{2}{*}{$\begin{array}{l}\text { Outcome } \\
\text { indicators }\end{array}$} \\
\hline & $T$ & $C$ & $T$ & $C$ & $T$ & $C$ & $T$ & $C$ & & \\
\hline $\begin{array}{l}\text { Zhang } \\
2020[13]\end{array}$ & 50 & 50 & $\begin{array}{l}24 / \\
26\end{array}$ & $\begin{array}{l}26 / \\
24\end{array}$ & $66.14 \pm 2.25$ & $66.28 \pm 2.33$ & $\begin{array}{c}\text { Control group + tongxinluo } \\
\text { capsule }\end{array}$ & Metformin & - & $\begin{array}{l}\text { (1) (2) (5) } \\
\text { (6) (7) }\end{array}$ \\
\hline $\begin{array}{l}\text { Zhang } \\
2020[14]\end{array}$ & 58 & 58 & $\begin{array}{c}39 / \\
19\end{array}$ & $\begin{array}{l}37 / \\
21\end{array}$ & $57.05 \pm 10.94$ & $56.46 \pm 10.37$ & $\begin{array}{l}\text { Control group + guilou } \\
\text { xiebai banxia and huangqi } \\
\text { xiaoke decoctions }\end{array}$ & $\begin{array}{l}\text { Metformin hydrochloride } \\
\text { sustained-release } \\
\text { tablets + isosorbide mononitrate } \\
\text { tablets }\end{array}$ & - & (2) (13) \\
\hline $\begin{array}{l}\text { Zhang } \\
2020[15]\end{array}$ & 52 & 52 & $\begin{array}{l}30 / \\
22\end{array}$ & $\begin{array}{l}28 / \\
24\end{array}$ & $51.6 \pm 8.2$ & $50.4 \pm 9.5$ & $\begin{array}{l}\text { Control group + danshen } \\
\text { ligustrazine injection }\end{array}$ & Standard western medicine & 2 weeks & (1) (7) \\
\hline $\begin{array}{l}\text { Zhang and } \\
\text { Zhang } \\
2020[16]\end{array}$ & 48 & 48 & $\begin{array}{c}30 / \\
18\end{array}$ & $\begin{array}{c}33 / \\
15\end{array}$ & $60.21 \pm 15.37$ & $59.87 \pm 14.65$ & $\begin{array}{l}\text { Control group + tongxinluo } \\
\text { capsule }\end{array}$ & $\begin{array}{c}\text { Isosorbide mononitrate } \\
\text { sustained-release } \\
\text { tablets }+ \text { aspirin enteric-coated } \\
\text { tablets + metoprolol } \\
\text { tartrate + valsartan } \\
\text { capsules + atorvastatin }\end{array}$ & 12 weeks & (7) (11) \\
\hline $\begin{array}{l}\text { Ye } 2020 \\
{[17]}\end{array}$ & 30 & 30 & $\begin{array}{l}14 / \\
16\end{array}$ & $\begin{array}{l}15 / \\
15\end{array}$ & $65.7 \pm 8.9$ & $65.2 \pm 7.6$ & $\begin{array}{l}\text { Control + buyang huanwu } \\
\text { decoction }\end{array}$ & $\begin{array}{l}\text { Calcium antagonists + anti- } \\
\text { platelet drugs }+\beta \text { receptor } \\
\text { blockers + nitrate drugs, lipid- } \\
\text { regulating drugs + angiotensin } \\
\text { converting enzyme inhibitors }\end{array}$ & 3 months & $\begin{array}{l}\text { (1) }(5)(6) \\
\text { (7) }(10)\end{array}$ \\
\hline $\begin{array}{l}\text { Xing et al. } \\
2019 \text { [18] }\end{array}$ & 100 & 100 & $\begin{array}{l}45 / \\
55\end{array}$ & $\begin{array}{c}42 / \\
58\end{array}$ & $53.01 \pm 2.52$ & $51.84 \pm 3.15$ & $\begin{array}{l}\text { Control group + tongxinluo, } \\
\text { ganlu xiaoke capsule }\end{array}$ & $\begin{array}{c}\text { Isosorbide dinitrate + aspirin } \\
\text { enteric-coated } \\
\text { tablets + atorvastatin }\end{array}$ & - & $\begin{array}{l}\text { (4) }(5)(6) \\
(14)\end{array}$ \\
\hline $\begin{array}{l}\text { Guan and } \\
\text { Shang } \\
2019[19]\end{array}$ & 39 & 39 & $\begin{array}{l}24 / \\
15\end{array}$ & $\begin{array}{l}22 / \\
17\end{array}$ & $54.5 \pm 2.9$ & $54.8 \pm 2.6$ & $\begin{array}{l}\text { Standard western } \\
\text { medicine + mai san }\end{array}$ & $\begin{array}{c}\text { Standard western } \\
\text { medicine + compound danshen } \\
\text { drop pills }\end{array}$ & 2 months & (1) (9) (12) \\
\hline $\begin{array}{l}\text { Liu et al. } \\
2019[20]\end{array}$ & 50 & 50 & $\begin{array}{l}34 / \\
16\end{array}$ & $\begin{array}{l}32 / \\
18\end{array}$ & $54.18 \pm 8.46$ & $53.66 \pm 9.12$ & $\begin{array}{l}\text { Control group }+ \text { shenqi } \\
\text { guilou xiebai banxia } \\
\text { decoction }\end{array}$ & $\begin{array}{c}\text { Metformin hydrochloride } \\
\text { sustained-release } \\
\text { tablets + isosorbide mononitrate } \\
\text { tablets }\end{array}$ & 45 days & (1) (7) (15) \\
\hline $\begin{array}{l}\text { Jiang } 2019 \\
{[21]}\end{array}$ & 35 & 35 & $\begin{array}{l}18 / \\
17\end{array}$ & $\begin{array}{l}19 / \\
16\end{array}$ & $3 / .2 \pm 7.4$ & 50.0 .0 .2 & $\begin{array}{l}\text { Standard western } \\
\text { medicine }+ \text { phlegm and } \\
\text { blood stasis decoction }\end{array}$ & $\begin{array}{c}\text { Standard } \\
\text { therapy + trimetazidine } \\
\text { hydrochloride }\end{array}$ & 4 weeks & $\begin{array}{l}\text { (1) }(2)(5) \\
(6)\end{array}$ \\
\hline $\begin{array}{l}\text { Zuo } 2019 \\
{[22]}\end{array}$ & 37 & 37 & $\begin{array}{r}21 / \\
16\end{array}$ & $\begin{array}{r}23 / \\
14\end{array}$ & $44.15 \pm 3.17$ & $43.93 \pm 3.04$ & + Shengmai San decoction & $\begin{array}{c}\text { Danshen dropping } \\
\text { pill + compound danshen } \\
\text { dropping pill }\end{array}$ & 2 months & $(5)(6)(13)$ \\
\hline $\begin{array}{l}\text { Liu et al. } \\
2019[23]\end{array}$ & 54 & 54 & $\begin{array}{l}28 / \\
26\end{array}$ & $\begin{array}{l}29 / \\
25\end{array}$ & $67.03 \pm 6.12$ & $66.78 \pm 5.81$ & $\begin{array}{c}\text { Control group + musk } \\
\text { yangxin san }\end{array}$ & $\begin{array}{c}\text { Sodium } \\
\text { trimetazidine }+ \text { isosorbide } \\
\text { mononitrate tablets }+ \text { aspirin } \\
\text { enteric-coated tablets }\end{array}$ & 3 month & $\begin{array}{l}\text { (1) (5) (6) } \\
\text { (7) (8) (16) }\end{array}$ \\
\hline
\end{tabular}


TABLE 1: Continued.

\begin{tabular}{|c|c|c|c|c|c|c|c|c|c|c|}
\hline \multirow{2}{*}{$\begin{array}{l}\text { Inclusion } \\
\text { studies }\end{array}$} & \multicolumn{2}{|c|}{$\begin{array}{l}\text { Sample } \\
\text { size }\end{array}$} & \multicolumn{2}{|c|}{$\begin{array}{l}\text { Male/ } \\
\text { female }\end{array}$} & \multicolumn{2}{|c|}{ Average age } & \multicolumn{2}{|c|}{ Intervention measures } & \multirow{2}{*}{$\begin{array}{l}\text { Treatment } \\
\text { course }\end{array}$} & \multirow{2}{*}{$\begin{array}{l}\text { Outcome } \\
\text { indicators }\end{array}$} \\
\hline & $T$ & $C$ & $T$ & C & $T$ & C & $T$ & $C$ & & \\
\hline o & 40 & 40 & $\begin{array}{c}21 / \\
19\end{array}$ & $\begin{array}{c}22 / \\
18\end{array}$ & $53.4 \pm 1.5$ & $52.5 \pm 1.3$ & $\begin{array}{c}\text { Control } \\
\text { group + deconstruction of } \\
\text { phlegm and removing blood } \\
\text { stasis }\end{array}$ & Standard western medicine & 1 month & $\begin{array}{l}\text { (1) }(5)(6) \\
\text { (17) }\end{array}$ \\
\hline Liu et al. & 35 & 35 & $\begin{array}{l}21 / \\
14\end{array}$ & $\begin{array}{c}19 / \\
16\end{array}$ & $46.35 \pm 2.36$ & $45.32 \pm 2.12$ & $\begin{array}{l}\text { Control group + yiqi shufeng } \\
\text { tongluo tang }\end{array}$ & Atorvastatin & - & (1) (3) (13) \\
\hline $\begin{array}{l}\text { Deng } 2018 \\
{[26]}\end{array}$ & 40 & 40 & $\begin{array}{c}21 / \\
19\end{array}$ & $\begin{array}{c}23 / \\
17\end{array}$ & $61.3 \pm 5.1$ & $60.1 \pm 5.4$ & $\begin{array}{l}\text { Antidiabetic drugs/ } \\
\text { insulin + long-acting } \\
\text { analgesic drugs + ziyin } \\
\text { huoxue decoction }\end{array}$ & $\begin{array}{c}\text { Antidiabetic drugs/ } \\
\text { insulin + long-lasting analgesic } \\
\text { drugs + compound danshen } \\
\text { dropping pills }\end{array}$ & 1 month & $\begin{array}{l}\text { (1) (4) (5) } \\
(6)(7)(14)\end{array}$ \\
\hline ng & 42 & 41 & $\begin{array}{c}23 / \\
19\end{array}$ & $\begin{array}{c}24 / \\
17\end{array}$ & $61.23 \pm 12.51$ & $61.42 \pm 12.38$ & $\begin{array}{l}\text { Control group }+ \text { danhong } \\
\text { injection }\end{array}$ & Standard western medicine & 2 weeks & $\begin{array}{l}\text { (1) }(2)(3) \\
\text { (5) }\end{array}$ \\
\hline Yan et al. & 35 & 35 & $\begin{array}{l}19 / \\
16\end{array}$ & $\begin{array}{c}20 / \\
15\end{array}$ & $1.1 \pm 12.9$ & $42.8 \pm 13.2$ & $\begin{array}{l}\text { Control group + yiqihua } \\
\text { turbid capsule }\end{array}$ & $\begin{array}{c}\text { Antidiabetic drugs, anti- } \\
\text { platelet, } \beta \text { receptor blockers, } \\
\text { statins }\end{array}$ & 3 months & $\begin{array}{l}\text { (1) (2) (5) } \\
\text { (6) }(13)\end{array}$ \\
\hline $\begin{array}{l}\text { Sui } 2016 \\
{[29]}\end{array}$ & 200 & 200 & $\begin{array}{c}100 / \\
100\end{array}$ & $\begin{array}{l}98 / \\
102\end{array}$ & $57.49 \pm 1.26$ & $57.5 \pm 1.25$ & $\begin{array}{l}\text { Antidiabetic drugs/ } \\
\text { insulin + long-acting } \\
\text { analgesic drugs + ziyin } \\
\text { huoxue decoction }\end{array}$ & $\begin{array}{r}\text { Antidiabetic } \\
\text { insulin + long-las } \\
\text { drugs + danshen }\end{array}$ & 1 months & ) $(7)$ \\
\hline $\begin{array}{l}\text { Tang } 2016 \\
{[30]}\end{array}$ & 55 & 55 & $\begin{array}{l}30 / \\
25\end{array}$ & $\begin{array}{l}32 / \\
23\end{array}$ & $56.15 \pm 1.54$ & $57.1 \pm 1.36$ & $\begin{array}{l}\text { Control group + ziyin } \\
\text { huoxue decoction. }\end{array}$ & $\begin{array}{c}\text { Antidiabetic } \\
\text { drugs + insulin + long-lasting } \\
\text { analgesic drugs }\end{array}$ & 1 month & (5) (6) (7) \\
\hline $\begin{array}{l}\text { Li and } \\
\text { Shang } \\
2015[31]\end{array}$ & 19 & 22 & $7 / 12$ & $\begin{array}{l}9 / \\
13\end{array}$ & $59.86 \pm 7.44$ & $61.21 \pm 7.38$ & $\begin{array}{l}\text { Control group + qishen yiqi } \\
\text { dropping pills }\end{array}$ & Standard western medicine & 8 weeks & $\begin{array}{l}(1)(2)(5) \\
(6)(7)\end{array}$ \\
\hline $\begin{array}{l}\text { He } 2013 \\
{[32]}\end{array}$ & 40 & 63 & - & - & - & - & $\begin{array}{l}\text { Antidiabetic drugs/ } \\
\text { insulin + long-acting } \\
\text { analgesic drugs + ziyin } \\
\text { huoxue decoction }\end{array}$ & $\begin{array}{c}\text { Antidiabetic drugs/ } \\
\text { insulin + long-acting analgesic } \\
\text { drugs + guanxin danshen } \\
\text { dropping pills }\end{array}$ & 1 month & $\begin{array}{l}\text { (1) (4) (5) } \\
\text { (6) }(7)\end{array}$ \\
\hline $\begin{array}{l}\text { Liu } 2012 \\
{[33]}\end{array}$ & 68 & 62 & $\begin{array}{l}46 / \\
22\end{array}$ & $\begin{array}{l}42 / \\
20\end{array}$ & & & $\begin{array}{l}\text { Conventional } \\
\text { treatment }+ \text { trimetazidine } \\
\text { hydrochloride }\end{array}$ & $\begin{array}{l}\text { Standard treatment }+ \text { phlegm- } \\
\text { dispelling and stasis removing } \\
\text { decoction }\end{array}$ & & $\begin{array}{l}\text { (1) }(2)(3) \\
\text { (4) }\end{array}$ \\
\hline $\begin{array}{l}\text { Li and } \\
\text { Yang } 2009 \\
{[34]}\end{array}$ & 36 & 36 & $\begin{array}{l}14 / \\
22\end{array}$ & $\begin{array}{l}11 / \\
25\end{array}$ & $59.5 \pm 8.96$ & $57.62 \pm 8.76$ & $\begin{array}{l}\text { Antidiabetic drugs/ } \\
\text { insulin + long-acting } \\
\text { analgesic drugs + ziyin } \\
\text { huoxue decoction }\end{array}$ & $\begin{array}{c}\text { Antidiabetic drugs/ } \\
\text { insulin + long-acting analgesic } \\
\text { drugs + compound danshen } \\
\text { dropping pills }\end{array}$ & 4 weeks & $\begin{array}{l}\text { (1) (4) (5) } \\
\text { (6) }(7)\end{array}$ \\
\hline $\begin{array}{l}\text { Ma } 2009 \\
{[35]}\end{array}$ & 30 & 30 & $\begin{array}{l}15 / \\
15\end{array}$ & $\begin{array}{l}15 / \\
15\end{array}$ & - & - & $\begin{array}{l}\text { Control group + yiqi tongluo } \\
\text { capsule }\end{array}$ & $\begin{array}{c}\text { Antidiabetic drugs/ } \\
\text { insulin }+ \text { isosorbide dinitrate } \\
\text { tablets }\end{array}$ & 1 month & $\begin{array}{l}\text { (1) }(4)(5) \\
(6)(13) \\
(14)\end{array}$ \\
\hline
\end{tabular}

$T$, observation group; $C$, control group; (1), total effective rate; (2), electrocardiogram effective rate; (3), adverse reaction; (4), angina pectoris attack times; (5), blood glucose; (6), 2-hour plasma glucose (2hPG); (7), blood lipid; (8), hemorheological index; (9), reactive protein; (10), interleukin 6; (11), glycosylated hemoglobin; (12), $\beta 2$-microglobulin; (13), TCM syndrome score; (14), angina pectoris pain duration; (15), vascular endothelial function; (16), coagulation function; (17), heart rate.

TABLE 2: Quality of included trials assessment.

\begin{tabular}{|c|c|c|c|c|c|}
\hline Inclusion studies & Randomized approach (2 points) & $\begin{array}{c}\text { Double-blind ( } 2 \\
\text { points) }\end{array}$ & Withdrawal loss (1 point) & Total & Article quality \\
\hline Zhang 2020 [13] & Random grouping method & Not described & No shedding & 1 & Low \\
\hline Zhang 2020 [14] & Not described & Not described & No shedding & 1 & Low \\
\hline Zhang 2020 [15] & Random allocation method & Not described & No shedding & 1 & Low \\
\hline Zhang and Zhang 2020 [16] & Random digital tables & Not described & No shedding & 3 & Medium \\
\hline Ye 2020 [17] & Random digital tables & Not described & No shedding & 3 & Medium \\
\hline Xing et al. 2019 [18] & Random digital tables & Not described & No shedding & 3 & Medium \\
\hline Guan and Shang 2019 [19] & Random digital tables & Not described & No shedding & 3 & Medium \\
\hline Liu et al. $2019[20]$ & Not described & Not described & No shedding & 1 & Low \\
\hline Jiang 2019 [21] & Not described & Not described & No shedding & 1 & Low \\
\hline Zuo 2019 [22] & Not described & Not described & No shedding & 1 & Low \\
\hline Liu et al. 2019 [23] & Not described & Not described & No shedding & 1 & Low \\
\hline Sun et al. 2018 [24] & Random digital tables & Not described & No shedding & 3 & Medium \\
\hline Liu et al. 2018 [25] & Not described & Not described & No shedding & 1 & Low \\
\hline
\end{tabular}


TABle 2: Continued.

\begin{tabular}{|c|c|c|c|c|c|}
\hline Inclusion studies & Randomized approach ( 2 points) & $\begin{array}{c}\text { Double-blind (2 } \\
\text { points) }\end{array}$ & Withdrawal loss (1 point) & Total & Article quality \\
\hline $\begin{array}{l}\text { Deng } 2018 \text { [26] } \\
\end{array}$ & Not described & Not described & No shedding & 1 & Low \\
\hline Zhang 2017 [27] & File sequence & Not described & No shedding & 1 & Low \\
\hline Yan et al. 2017 [28] & Random digital tables & Not described & No shedding & 3 & Medium \\
\hline Sui 2016 [29] & Not described & Not described & No shedding & 1 & Low \\
\hline Tang 2016 [30] & Not described & Not described & No shedding & 1 & Low \\
\hline $\mathrm{Li}$ and Shang 2015 [31] & Random digital tables & Not described & No shedding & 3 & Medium \\
\hline He 2013 [32] & Random digital tables & Not described & No shedding & 3 & Medium \\
\hline Liu 2012 [33] & Not described & Not described & No shedding & 1 & Low \\
\hline $\mathrm{Li}$ and Yang 2009 [34] & Not described & Not described & No shedding & 1 & Low \\
\hline Ma 2009 [35] & Not described & Not described & No shedding & 1 & Low \\
\hline
\end{tabular}

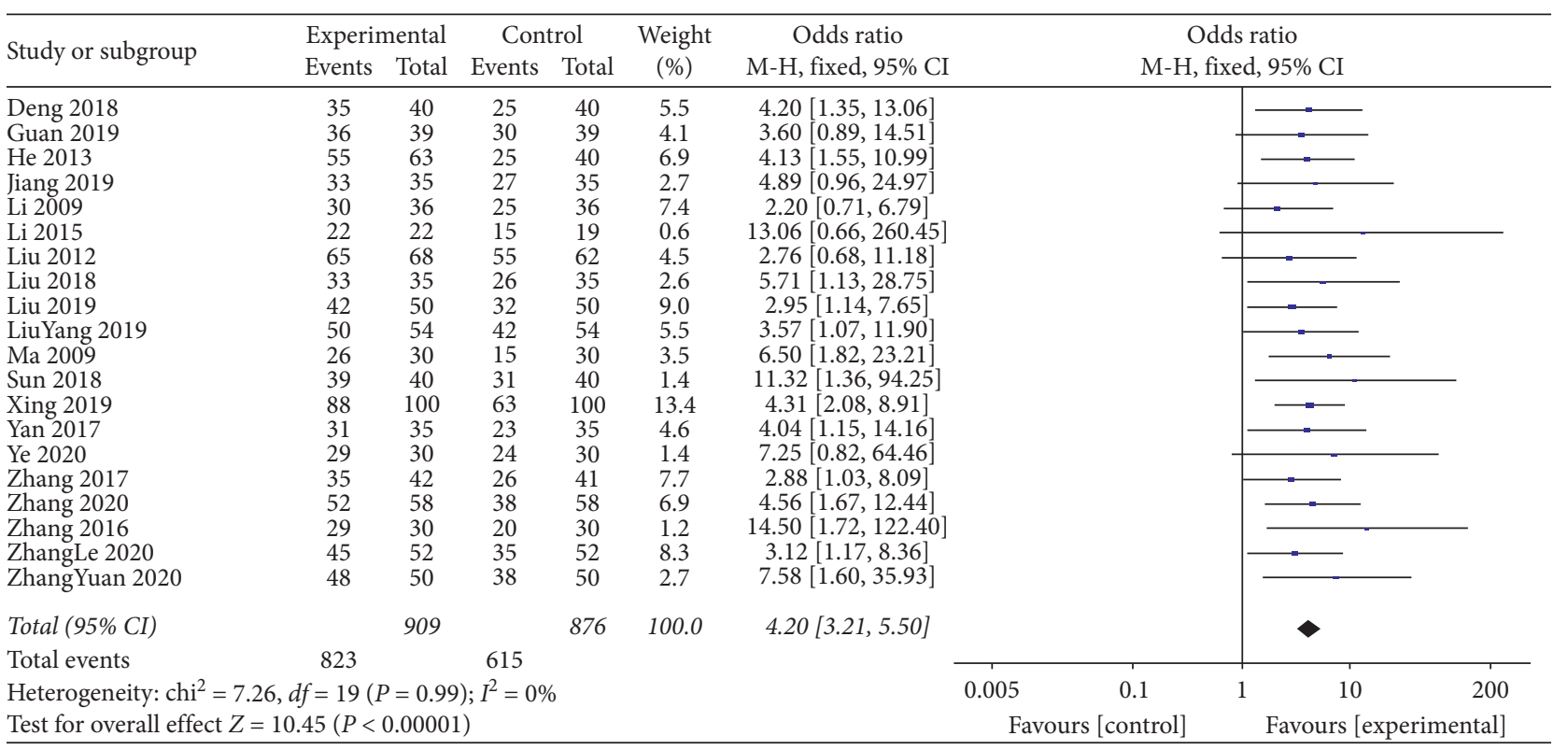

Figure 2: Meta-analysis of total clinical efficiency.

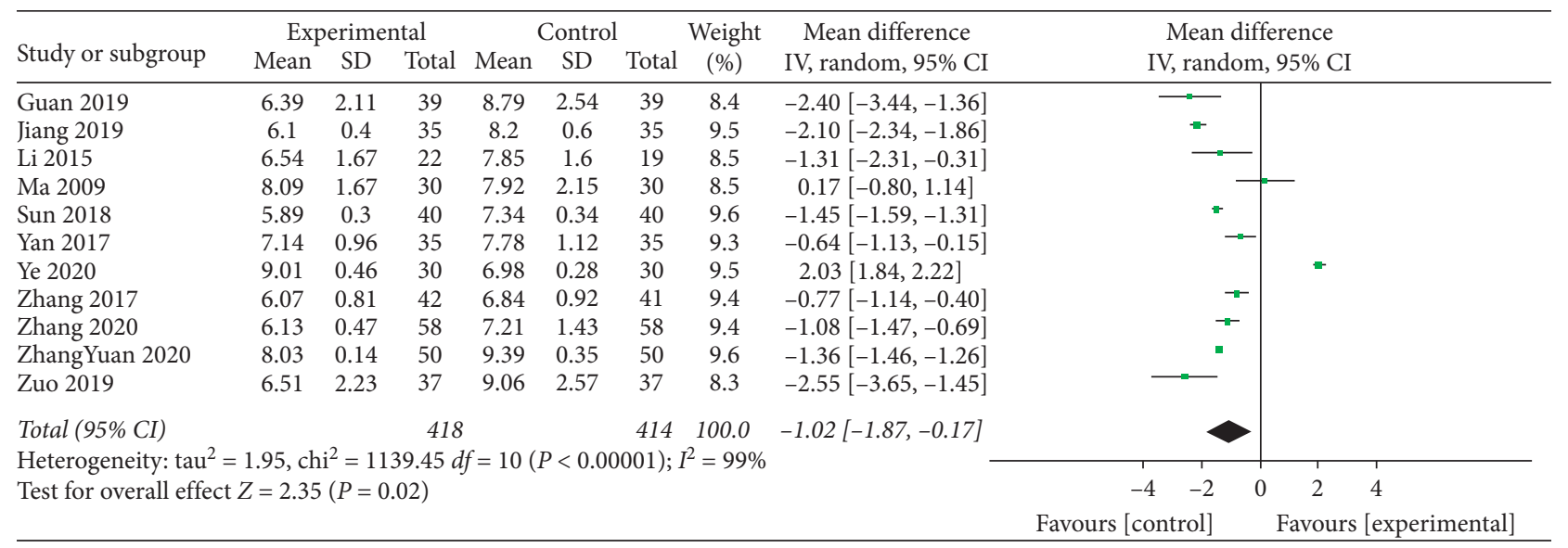

FIGURE 3: Meta-analysis of fasting blood glucose levels. 


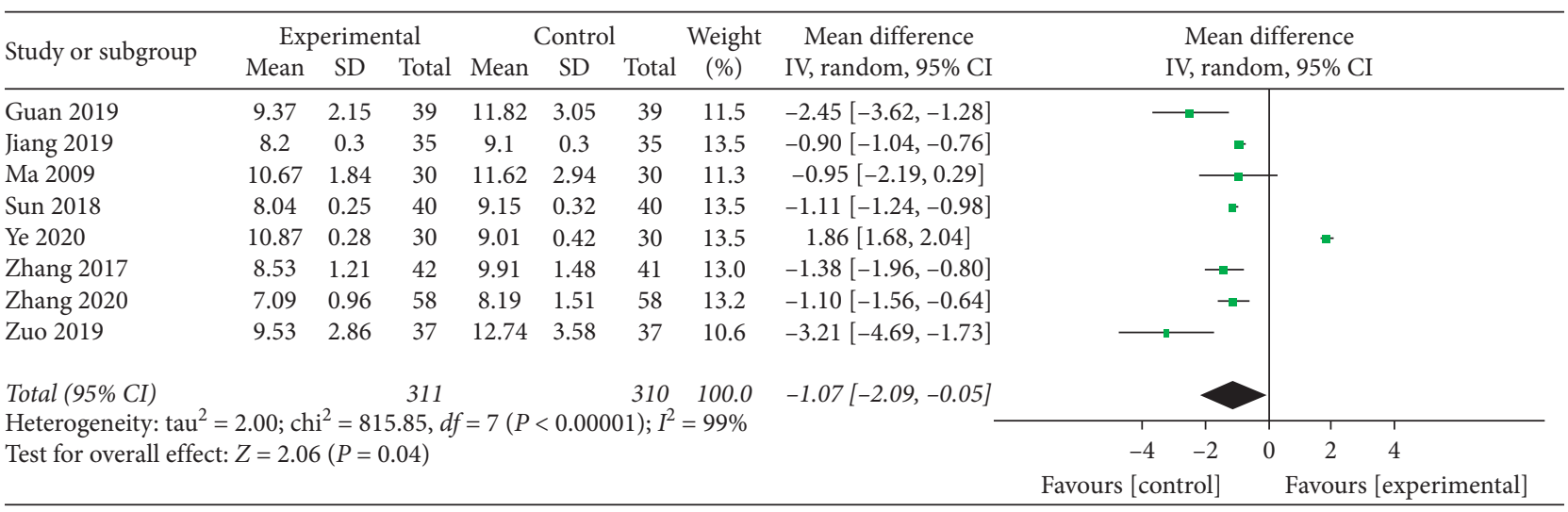

FIgURE 4: Two-hour plasma glucose (2hPG) levels.

\begin{tabular}{|c|c|c|c|c|c|c|c|c|c|c|}
\hline \multirow{3}{*}{$\begin{array}{l}\text { Study or subgroup } \\
\text { Deng } 2018\end{array}$} & \multicolumn{3}{|c|}{ Experimental } & \multicolumn{3}{|c|}{ Control } & \multirow{2}{*}{$\begin{array}{l}\text { Weight } \\
(\%)\end{array}$} & \multirow{2}{*}{$\begin{array}{l}\text { Mean difference } \\
\text { IV, random, } 95 \% \mathrm{CI}\end{array}$} & \multirow{2}{*}{\multicolumn{2}{|c|}{$\begin{array}{l}\text { Mean difference } \\
\text { IV, random, } 95 \% \text { CI }\end{array}$}} \\
\hline & Mean & $\mathrm{SD}$ & Total & Mean & $\mathrm{SD}$ & Total & & & & \\
\hline & 4.09 & 0.83 & 40 & 6.14 & 0.54 & 40 & 7.8 & $-2.05[-2.36,-1.74]$ & - & \\
\hline He 2013 & 4.13 & 0.74 & 63 & 6.11 & 0.72 & 40 & 7.9 & $-1.98[-2.27,-1.69]$ & $\rightarrow$ & \\
\hline Li 2009 & 4.11 & 0.85 & 36 & 6.12 & 0.56 & 36 & 7.7 & $-2.01[-2.34,-1.68]$ & $\rightarrow$ & \\
\hline Li 2015 & 4.89 & 0.64 & 22 & 5.76 & 0.7 & 19 & 7.4 & $-0.87[-1.28,-0.46]$ & $=-$ & \\
\hline Liu 2019 & 3.2 & 0.79 & 50 & 3.68 & 0.91 & 50 & 7.7 & $-0.48[-0.81,-0.15]$ & $=$ & \\
\hline LiuYang 2019 & 4.32 & 0.91 & 54 & 6.81 & 1.02 & 54 & 7.6 & $-2.49[-2.85,-2.13]$ & - & \\
\hline Sui 2016 & 5.45 & 0.19 & 200 & 5.75 & 0.26 & 200 & 8.4 & $-0.30[-0.34,-0.26]$ & - & $=$ \\
\hline Tang 2016 & 5.42 & 0.18 & 55 & 5.73 & 0.28 & 55 & 8.3 & $-0.31[-0.40,-0.22]$ & - & $=$ \\
\hline Ye 2020 & 3.65 & 1.64 & 30 & 4.45 & 1.42 & 30 & 5.7 & $-0.80[-1.58,-0.02]$ & - & \\
\hline Zhang 2020 & 4.29 & 0.91 & 58 & 4.96 & 1.13 & 58 & 7.5 & $-0.67[-1.04,-0.30]$ & $\rightarrow$ & \\
\hline ZhangLe 2020 & 4.21 & 1.01 & 52 & 5.97 & 0.94 & 52 & 7.5 & $-1.76[-2.14,-1.38]$ & - & \\
\hline ZhangTing 2020 & 3.49 & 0.3 & 48 & 3.76 & 0.29 & 48 & 8.3 & $-0.27[-0.39,-0.15]$ & $=$ & - \\
\hline ZhangYuan 2020 & 5.33 & 0.42 & 50 & 6.46 & 0.44 & 50 & 8.2 & $-1.13[-1.30,-0.96]$ & - & \\
\hline Total (95\% CI) & & & 758 & & & 732 & 100.0 & $-1.16[-1.48,-0.83]$ & $\diamond$ & \\
\hline \multirow{2}{*}{\multicolumn{9}{|c|}{$\begin{array}{l}\text { Heterogeneity: } \operatorname{tau}^{2}=0.32 ; \mathrm{chi}^{2}=594.86, d f=12(P<0.00001) ; I^{2}=98 \% \\
\text { Test for overall effect: } Z=7.00(P<0.00001)\end{array}$}} & -2 & 0 \\
\hline & & & & & & & & & Favours [control] & Favours [experimental] \\
\hline
\end{tabular}

FIgURE 5: Meta-analysis of total cholesterol levels.

were statistically heterogeneous $\left(I^{2}=51 \% ; \quad P=0.02\right)$. Therefore, the meta-analysis was performed using the random effects model. The addition of TCM compounds to standard treatments resulted in a beneficial effect $(\mathrm{MD}=0.19 ; 95 \% \mathrm{CI}, 0.12$ to $0.26 ; P=0.0001$; Figure 8$)$.

3.4.8. Effect on Electrocardiogram. Electrocardiograms were reported for a total of 556 participants across 6 studies with no statistical between-study heterogeneity $\left(I^{2}=22 \%\right.$; $P=0.27)$. Therefore, the meta-analysis of the data was performed using the fixed effect model. The addition of TCM compounds to standard treatments resulted in a beneficial effect on the electrocardiograms ( $\mathrm{OR}=4.20$; 95\% CI, 3.15 to 8.18; $P=0.0001$; Figure 9).

3.4.9. Safety Assessment of TCM Compounds. Safety assessment of TCM compounds was performed in 3 studies with no statistical between-study heterogeneity $\left(I^{2}=14 \%\right.$; $P=0.31$ ) including a total of 283 participants. A metaanalysis of the data from these three studies was performed using the fixed effect model. The use of TCM compounds combined with standard treatments was not associated with significant difference in the adverse effects recorded $(\mathrm{OR}=0.53 ; 95 \% \mathrm{CI}, 0.19$ to $5.50 ; P=0.21$; Figure 10).

3.4.10. Publication Bias Analysis. Publication bias on the outcome of clinical efficiency and electrocardiograms in the publications included in this study was analyzed according to the pooled qualitative (funnel plot) method. Using the log (OR) as boundary, the studies were asymmetrically distributed between the left- and right-hand sides, indicating a publication bias. (Figure 11).

\section{Discussion}

With lifestyle changes, urbanization and aging of the population, cardiovascular diseases, and cancer have become the leading causes of death in Chinese adults [10]. The prevalence of DM and prediabetes in Chinese adults are $9.7 \%$ and $15.5 \%$, respectively. $\mathrm{CHD}$ and $\mathrm{DM}$ have become major public health problems. There is an interaction between DM 


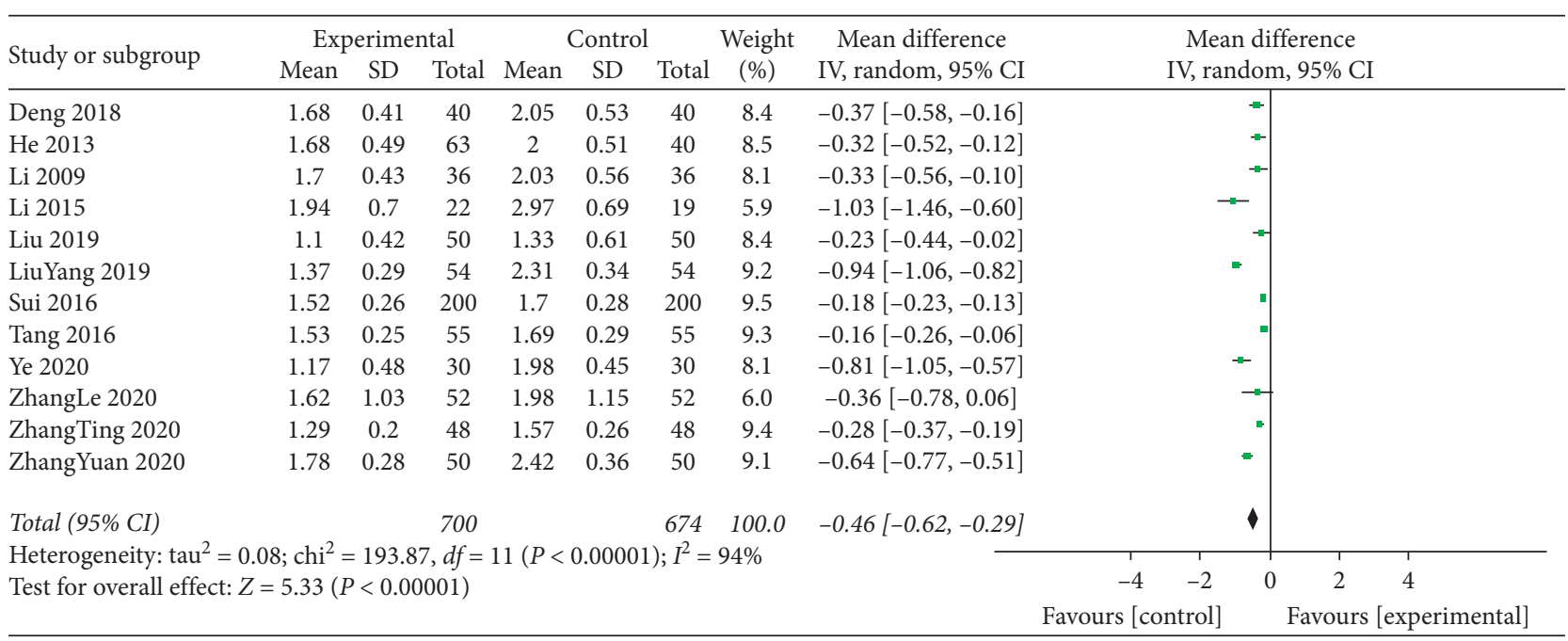

FIGURE 6: Meta-analysis of triglyceride levels.

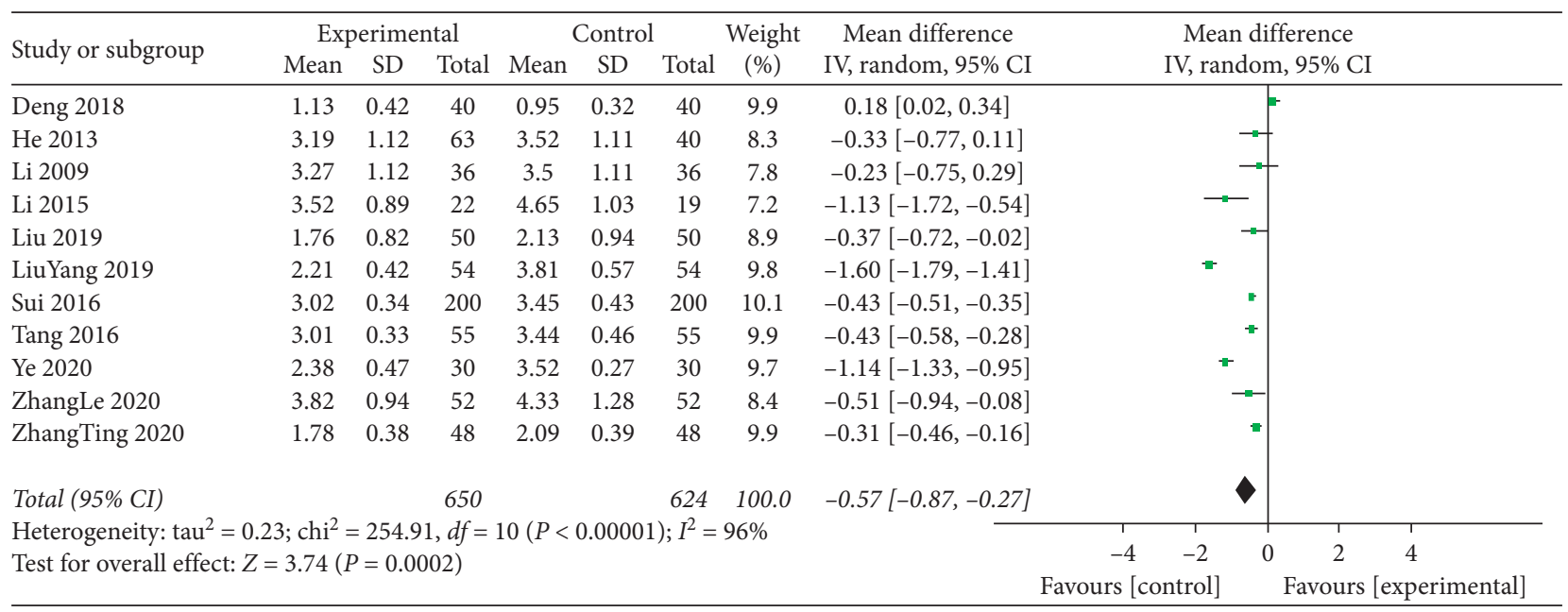

FIGURE 7: Meta-analysis of low-density lipoprotein cholesterol (LDL-C) levels.

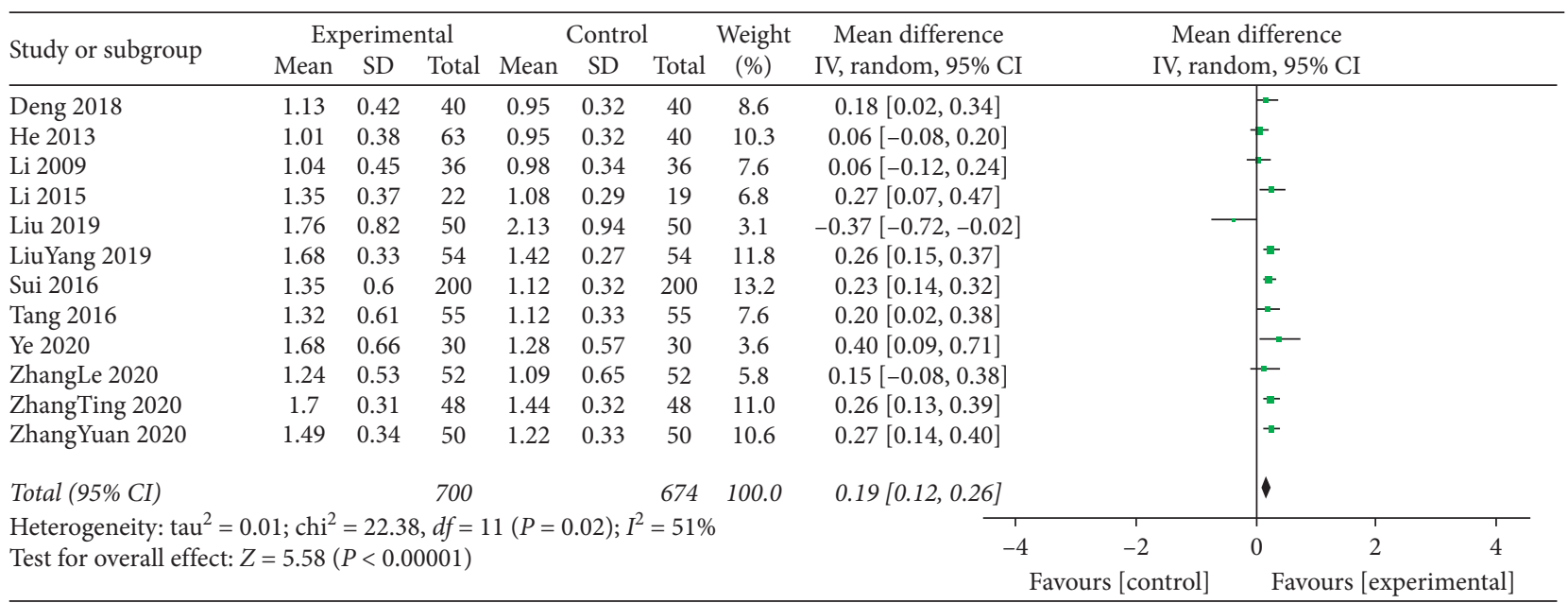

FIGURE 8: Meta-analysis of high-density lipoprotein cholesterol (HDL-C) levels. 


\begin{tabular}{|c|c|c|c|c|c|c|c|c|c|}
\hline \multirow{2}{*}{ Study or subgroup } & \multicolumn{2}{|c|}{ Experimental } & \multicolumn{2}{|c|}{ Control } & \multirow{2}{*}{$\begin{array}{l}\text { Weight } \\
(\%)\end{array}$} & \multirow{2}{*}{$\begin{array}{c}\text { Odds ratio } \\
\mathrm{M}-\mathrm{H} \text {, fixed, } 95 \% \mathrm{CI}\end{array}$} & \multirow{2}{*}{\multicolumn{2}{|c|}{$\begin{array}{c}\text { Odds ratio } \\
\text { M-H, fixed, 95\% CI }\end{array}$}} & \\
\hline & Events & Total & Events & Total & & & & & \\
\hline Li 2009 & 30 & 36 & 25 & 36 & 24.7 & $2.20[0.71,6.79]$ & & \multicolumn{2}{|l|}{$\longrightarrow$} \\
\hline Li 2015 & 22 & 22 & 14 & 19 & 2.0 & $17.07[0.88,332.49]$ & & & \\
\hline Ma 2009 & 29 & 30 & 14 & 30 & 2.8 & $33.14[3.98,275.73]$ & & \multirow{2}{*}{$\rightarrow$} & \\
\hline Xing 2019 & 88 & 100 & 63 & 100 & 44.9 & $4.31[2.08,8.91]$ & & & \\
\hline Zhang 2017 & 38 & 42 & 29 & 41 & 16.6 & $3.93[1.15,13.46]$ & & $\longrightarrow$ & \\
\hline ZhangYuan 2020 & 48 & 50 & 38 & 50 & 9.0 & $7.58[1.60,35.93]$ & & & \\
\hline Total $(95 \%$ CI) & \multicolumn{2}{|c|}{280} & & 276 & 100.0 & $5.07[3.15,8.18]$ & & & \\
\hline \multicolumn{5}{|c|}{$\begin{array}{l}\text { Total events } \\
\text { Heterogeneity: } \mathrm{chi}^{2}=6.38, d f=5(P=0.27) ; I^{2}=22 \%\end{array}$} & & & & & \\
\hline \multicolumn{5}{|c|}{ Test for overall effect: $Z=6.67(P<0.00001)$} & & 0.002 & 0.1 & $1 \quad 10$ & 500 \\
\hline & & & & & & \multicolumn{2}{|c|}{ Favours [control] } & Favours [ & al] \\
\hline
\end{tabular}

FIgURE 9: Meta-analysis of the effects on the electrocardiogram.

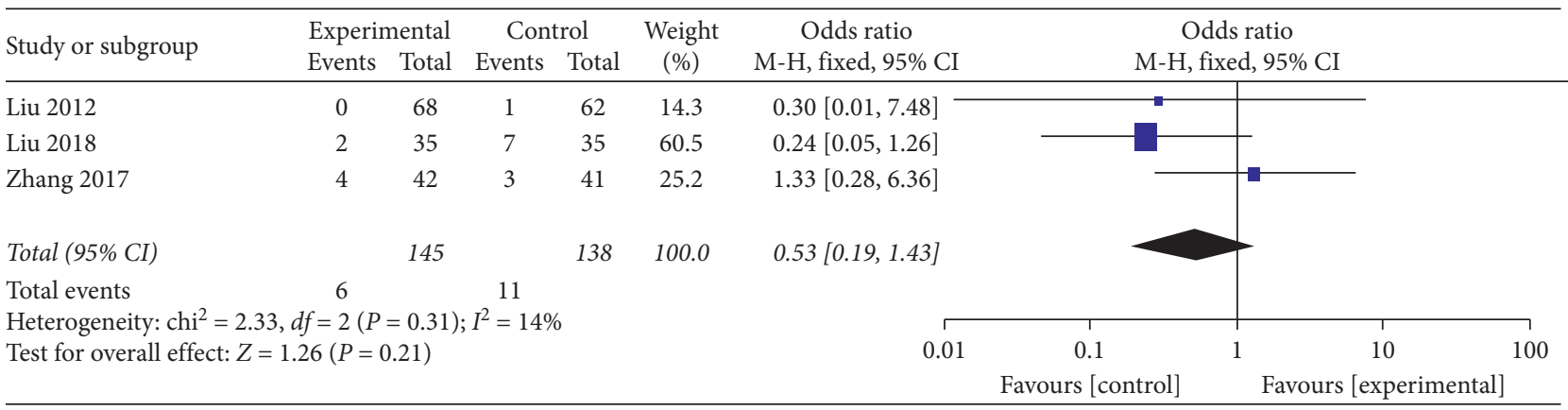

FIgURE 10: Meta-analysis of adverse reactions.
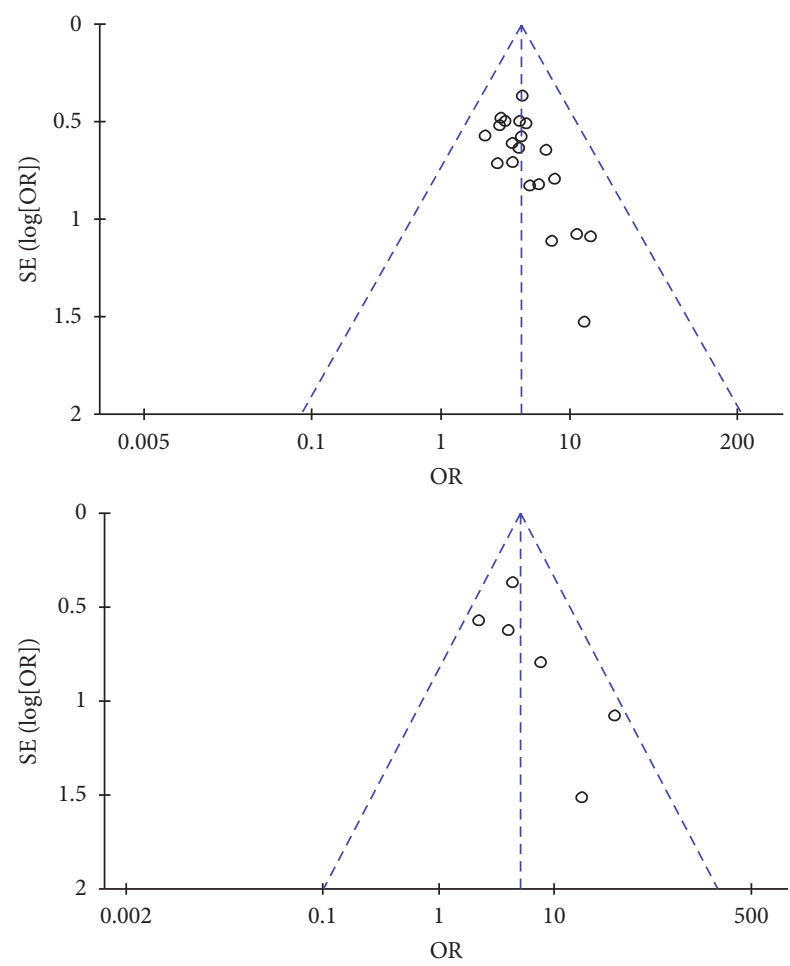

FIGURE 11: Analysis of publication bias of clinical total effective rate and electrocardiogram effective rate. and cardiovascular diseases. The risk of cardiovascular events in diabetic patients is nearly twice as high as in nondiabetic patients [36]. Data showed that about $75 \%$ of the deaths occurring in DM patients are associated with coronary artery diseases [37]. The results of the European Heart Survey also showed that about two-thirds of the CHD cases are associated with glucose metabolism disorders. Therefore, the treatment of DM complicated by CHD is an important issue.

Standard treatments mostly included oral hypoglycemic drugs or insulin to control blood glucose levels in addition to the management of lifestyle factors, such as diet, exercise, and weight control. However, DM requires long-term continuous control, patient compliance is poor, and the results of these therapeutic strategies are not satisfactory. $\mathrm{DM}$ with $\mathrm{CHD}$ is classified in the "Xiaoke" and "chest arthralgia" category in TCM theory. The disease is mainly caused by Qi Yin deficiency. Qi and blood stasis caused by the heart pulse are insuperable. Qi deficiency leads to weak blood flow, blood stasis, long-term heat accumulation, and heat injury, provoking further inside blood stasis [38]. Western medicine uses nitrates for the treatment of $\mathrm{CHD}$ symptoms, but drug resistance occurs during the treatment of DM, when the use of hypoglycemic drugs increases the load on the heart. The combination of these two types of drugs, which represent an almost life-long treatment, 
seriously reduces patients' quality of life [39]. Many studies have shown that Chinese herbal compounds can be used to successfully treat CHD. These not only improve clinical efficiency but also relieve FBG, total cholesterol, triglyceride, LDL-C, and 2hPG levels, and other DM indices to some extent.

The meta-analyses we performed showed some limitations of the evaluation system: (1) some publication bias was detected; (2) the overall quality of the included studies was low; (3) there was a lack of objective unified criteria for the determination of clinical efficacy; (4) the control groups received different treatments; and (5) all the studies were published in Chinese.

The results of the analysis could be biased. Although there are some limitations in TCM due to the lack of basic research, evidence-based practices that may be effective make it an attractive treatment system for many diseases. The accuracy of the meta-analysis results depends on the article selection. Therefore, the requirements for future experiments should be multicenter, large sample, randomized, control, and double-blind allocation hidden experiment. These requirements suggest that clinical trials have to further improve their scientific value, strictly control the implementation of standards, provide clearer and more detailed descriptions of randomization, blind test, and more particularly, improve the quality of the testing methodology and standardize the clinical RCT reports, such that they can provide more effective information for clinical applications.

\section{Conclusion}

Of the 23 publications included in this study, 20 reported information on clinical comprehensive efficiency, 11 reported information on FBG levels, 13 reported information on total cholesterol levels, 12 reported information on triglyceride levels, 11 reported information on LDL-C levels, and 11 reported information on HDL-C levels. All of these parameters were statistically improved by the use of TCM compounds.

In summary, based on current evidence, we conclude that combined therapy could increase the clinical total effective rate and reduce $\mathrm{FBG}$, total cholesterol, and HDL-C levels. There was no evidence showing that combination therapy would lead to safety problems. A number of sensitivity analyses have shown that our conclusions are robust.

After evaluating the quality of the articles included in the present meta-analysis, we found that most articles were of low quality, which is the main limitation of our study. International methodologies and rigorous RCTs can produce better tests. Therefore, in order to evaluate the clinical efficacy of TCM and provide a strong scientific basis for the development of TCM, more high-quality studies are needed to provide more reliable data for metaanalysis.
Abbreviations
AE: $\quad$ Adverse event
CHD: Coronary heart disease
DM: Diabetes mellitus
FBG: $\quad$ Fasting blood glucose
HDL-C: High-density lipoprotein-cholesterol
LDL-C: Low-density lipoprotein-cholesterol
MD: $\quad$ Mean difference
OR: Odds ratio
RCT: Randomized controlled trial
TCM: Traditional Chinese medicine
TER: Total efficiency rate
T2DM: Type 2 diabetes mellitus
2Hpg: 2-Hour plasma glucose.

\section{Data Availability}

All the data generated or analyzed during this study are included in this published article.

\section{Conflicts of Interest}

The authors declare that they have no conflicts of interest.

\section{Authors' Contributions}

Mengqi Yang, Min Wang, and Zhishu Tang designed the study and provided general guidance for the drafting of the protocols. Mengqi Yang and Zhongxing Song drafted the protocol. Min Wang, Zhishu Tang, and Zhongxing Song designed the search strategy. Mengqi Yang, Chunli Cui, and Min Wang drafted the manuscript. Chunli Cui, Min Cheng, and Yanru Liu reviewed and revised the manuscript. All the authors have read and approved the final version of the manuscript.

\section{Acknowledgments}

The authors thank LetPub (http://www.letpub.com) for its linguistic assistance during the preparation of this manuscript. This project was supported by the National Science and Technology Major Project grant (2019ZX09301-133); the National Modern Agricultural Industrial Technology System Construction Special Project grant (CARS-21); and a key project grant obtained from the Central Government: the ability establishment of sustainable use for valuable Chinese medicine resources (2060302). 


\section{References}

[1] H. J. Yuan, S. Y. Lin, G. J. Zhang et al., "New thoughts on material basis and mechanism of Yin-tonifying traditional Chinese medicine in treatment of diabetes mellitus and its complications," China Journal of Chinese Materia Medica, vol. 45 , no. 3, pp. 531-538, 2020.

[2] W. Zhong, "Clinical observation on treatment of 38 cases of diabetic complicated with coronary heart disease by traditional Chinese medicine," Chinese Journal of Ethnomedicine and Ethnopharmacy, vol. 23, no. 8, p. 83, 2014.

[3] D. Fang, X. Deng, Z. H. He, J. B. Wu, and M. Luo, "Altered levels and clinical significance of serum miR-24 as a potential biomarker in diabetes mellitus with coronary heart disease," Chinese Journal of Arteriosclerosis, vol. 26, no. 5, pp. 491-496, 2018.

[4] S. Wu, Y. Liu, and Q. Song, "Multivariate analysis of risk and prognostic factors in patients with coronary heart disease," Chinese General Practice, vol. 21, no. 29, pp. 3562-3570, 2018.

[5] X. L. Zhou, Y. M. Zhang, F. Wu, and J. P. Zhao, "Detection and drug resistance of extended spectrum $\beta$-lactamase-producing bacteria," Chinese Journal of Nosocomiology, vol. 22, no. 7, pp. 1489-1491, 2012.

[6] J. Li and L. Song, "Effect of sugar collaterals detoxification combined with western medicine in treatment of diabetes complicated with angina pectoris," Chinese Archives of Traditional Chinese Medicine, vol. 37, no. 2, pp. 505-508, 2019.

[7] Z. Yang, X. Bao, C. Dong, and Y. Gong, "Study progress on TCM syndrome differentiation of type 2 diabetic mellitus combined with coronary heart disease," Global Traditional Chinese Medicine, vol. 10, no. 5, pp. 631-635, 2017.

[8] H. Y. Ding, "Treatment of blood circulation Yiqiyangyin 79 cases of angina pectoris," Journal of Practical Traditional Chinese Internal Medicine, vol. 25, no. 4, pp. 70-71, 2011.

[9] S. Sun, H. Huang, C. Huang, and M. Liu, "Literature study on prescription regularity in the treatment of diabetes mellitus complicated with coronary heart disease," Journal of Traditional Chinese Medicine, vol. 59, no. 14, pp. 1236-1240, 2018.

[10] Y. X. Zhang, Y. B. Hu, H. F. Yao, and J. Wang, "Effects of Yiqiyangyinghuoxuejiedu Decoction on the clinical effect, CRP and HCY in patients with diabetes mellitus with coronary heart disease," Journal of Emergency in Traditional Chinese Medicine, vol. 20, no. 8, pp. 1218-1219, 2011.

[11] Y. W. Zou, "Efficacy observation of Yixinshu Capsule treatment of type 2 diabetes combined heart disease," Journal of Traditional Chinese Medicine University of Hunan, vol. 33, no. 8, pp. 11-12, 2013.

[12] Y. W. Zou, "Ping Xiaotong Xin Capsule treatment type 2 diabetes with coronary heart disease angina pectoris curative effect observation," Shandong Journal of Traditional Chinese Medicine, vol. 33, no. 4, pp. 266-269, 2014.

[13] Y. Zhang, "Effect of Tongxinluo Capsule on angina pectoris of type 2 diabetes complicated with coronary heart disease," Diabetes New World, vol. 23, no. 6, pp. 81-83, 2020.

[14] Y. X. Zhang, "Clinical effect of Guilou Xiebai Banxia Decoction and Huangqi Xiaoke Decoction combined with western medicine on coronary heart disease complicated with diabetes mellitus," Modern Diagnosis \& Treatment, vol. 31, no. 1, pp. 23-24, 2020.

[15] L. Zhang, "Clinical efficacy of salvia miltiorrhiza ligustrazine in the treatment of diabetic patients with coronary heart disease," Guide of China Medicine, vol. 18, no. 3, pp. 171-172, 2020 .
[16] T. Zhang and C. Zhang, "Effect of Tongxinluo Capsule on clinical outcomes of patients with coronary heart disease complicated with diabetes," Continuing Medical Education, vol. 34, no. 5, pp. 167-168, 2020.

[17] M. Ye, "Exploration on the effect of Buyang Huanwu Tang combined with health education in intervening coronary heart disease complicated with diabetes," Journal of New Chinese Medicine, vol. 52, no. 5, pp. 149-153, 2020.

[18] X. Xing, W. Zhang, and H. Zhang, "Effect of Tongxinluo Capsule and Ganlu Xiaoke Capsule combined with routine treatment of western medicine for coronary heart disease complicated with diabetes," Journal of Clinical Medicine in Practice, vol. 23, no. 12, pp. 68-71, 2019.

[19] J. Guan and X. Shang, "Prognostic effect of Shengmai San Decoction on Qi-yin deficiency syndrome diabetes mellitus complicated with coronary heart disease angina pectoris," Clinical Research, vol. 27, no. 11, pp. 134-135, 2019.

[20] X. Liu, T. Li, H. Li, and H. Liu, "Treatment of 50 cases of coronary atherosclerotic heart disease with diabetes mellitus with liver and kidney Yin deficiency by Shenqi Guilou Xiebai Banxia Decoction," Global Traditional Chinese Medicine, vol. 12, no. 12, pp. 1871-1874, 2019.

[21] X. Y. Jiang, "Effect of Diantuan Quyu Decoction on clinical treatment of diabetes mellitus with coronary heart disease," Nei Mongol Journal of Traditional Chinese Medicine, vol. 38, no. 2, pp. 12-13, 2019.

[22] Y. Zuo, "Clinical observation of Shengmai San Decoction for treatment of diabetes mellitus with coronary heart disease and angina pectoris," Chinese Journal of Clinical Rational Drug Use, vol. 12, no. 35, pp. 34-35, 2019.

[23] Y. Liu, J. Han, and Q. Wang, "Effect of Shexiang Yangxin powder on diabetic mellitus complicated with coronary atherosclerotic heart disease and its influence on blood lipid ,hemorheological index and coagulation function," Shaanxi Journal of Traditional Chinese Medicine, vol. 40, no. 5, pp. 576-579, 2019.

[24] H. Sun, P. Wang, and J. Su, "Observation on the curative effect of self-made Tang-Du-Tang-Quyu decoction on diabetes mellitus complicated with coronary heart disease," Diabetes New World, vol. 21, no. 2, pp. 43-44, 2018.

[25] Z. Liu, Z. Wang, and X. Wang, "Observation on curative effect of Yiqi Shufeng Tongluo prescription combined with atorvastatin in treating of type 2 diabetes mellitus complicated with coronary heart disease," China Journal of Chinese Medicine, vol. 33, no. 5, pp. 751-755, 2018.

[26] X. S. Deng, "Clinical research on associating invigorating yin and promoting blood flow in the treatment of diabetes complicated with coronary atherosclerotic heart disease," China Practical Medical, vol. 13, no. 29, pp. 85-87, 2018.

[27] H. Zhang, "Effect of Danhong injection and rabeprolol hydrochloride on coronary heart disease and diabetes mellitus," Henan Medical Research, vol. 26, no. 24, pp. 4471-4472, 2017.

[28] D. Yan, X. Guo, Y. Liu, and J. Guo, "Clinical observation on Yiqi Huazhuo Capsules in the treatment of diabetes mellitus complicated with coronary heart disease," Chinese Journal of Integrative Medicine on Cardio/Cerebrovascular Disease, vol. 15, no. 16, pp. 1956-1958, 2017.

[29] D. H. Sui, "Ziyin Huoxue method in treatment of coronary atherosclerosis diabetic heart disease clinical curative effect observation," Diabetes New World, vol. 19, no. 7, pp. 76-77, 2016.

[30] B. Tang, "Clinical analysis of nourishing yin and activating blood circulation in treating diabetic patients with coronary 
atherosclerotic heart disease," Health Care Today, vol. 16, no. 10, p. 117, 2016.

[31] X. Li and G. Shang, "Clinical study of Qishen Yiqu dropping pills for coronary heart disease complicated with diabetes mellitus," Forum on Traditional Chinese Medicine, vol. 30, no. 4, pp. 25-26, 2015.

[32] D. He, "Clinical analysis of nourishing yin and activating blood circulation in treating diabetic patients with coronary atherosclerotic heart disease," China Foreign Medical Treatment, vol. 32, no. 12, p. 92, 2013.

[33] G. Liu, "Observation on the effect of self-drafting deconstruction and dispelling of blood stasis on 68 cases of diabetes complicated with coronary heart disease," Chinese Community Doctors, vol. 14, no. 23, p. 167, 2012.

[34] C. Li and L. Yang, "Treatment of coronary atherosclerotic heart disease with diabetes mellitus," Chinese Primary Health Care, vol. 23, no. 7, pp. 100-101, 2009.

[35] Z. Ma, "Effect of Yiqitongluo capsule on the treatment of diabetes mellitus complicated by coronary heart disease and angina pectoris," Hebei Journal of Traditional Chinese Medicine, vol. 31, no. 7, pp. 974-976, 2009.

[36] R. N. Bai, R. X. Xi, and L. Z. Li, "Researches of coronary heart disease complicated with diabetes mellitus:a review of recent progress in the domain of Chinese medicine," Global Traditional Chinese Medicine, vol. 7, no. 5, pp. 389-392, 2014.

[37] D. Wang, T. Y. Chen, K. Zhou, and F. J. Liu, "Effect analysis between glycated albumin and glycosylated hemoglobin on coronary artery disease in patients with type 2 diabetes mellitus," Medical \& Pharmaceutical Journal of Chinese People's Liberation Army, vol. 30, no. 3, pp. 31-34, 2018.

[38] L. Liu, X. Shan, C. Sun, and S. Geng, "Preliminary study on therapeutic effect and mechanism of using Yangxin Yiqi Decoction in the treatment of type 2 diabetes mellitus complicated with coronary heart disease," Journal of Sichuan Traditional Chinese Medicine, vol. 37, no. 8, pp. 80-83, 2019.

[39] Y. Li, "Systematic evaluation of traditional Chinese medicine in the treatment of coronary heart disease with diabetes mellitus," Nei Mongol Journal of Traditional Chinese Medicine, vol. 36, no. 9, pp. 84-85, 2017. 